Abstract

\title{
New Explanation for the Mpemba Effect ${ }^{\dagger}$
}

\section{Ilias Tyrovolas}

Ministry of Rural Development \& Food, Nafplio, Greece

† Presented at the Entropy 2021: The Scientific Tool of the 21st Century, 5-7 May 2021; Available online: https://sciforum.net/conference/Entropy2021/.

Published: 5 May 2021

The purpose of this study is to check out the involvement of entropy in Mpemba effect. Provided that preheating of the water the cooling duration is reduced, we theoretically show that water gains more entropy when warmed and re-cooled to the original temperature. Water molecules are oriented dipoles joined by hydrogen bonds. When water is heated, this structure collapses (i.e., the entropy increases). When water is re-cooled to a lower temperature, the previous structure is not re-formed immediately. Sometimes, when the re-cooling is performed within a freezer, there is not enough time for the structure to re-form because of the high cooling rate. The entropy reduction curve as a function of the temperature, $S=f(T)$, shows retardation (a lag) relative to the entropy growth curve. Water that has been heated and re-cooled to the initial temperature shows greater entropy than that before it was heated. This means that, while its molecules now have the same kinetic energy, their thermal motion after heating is less oriented with respect to the structure mentioned above. After re-cooling, random collisions are more likely, owing to this the temperature decreases more quickly.

(C) 2021 by the author. Licensee MDPI, Basel, Switzerland. This article is an open access article distributed under the terms and conditions of the Creative Commons Attribution (CC BY) license (http://creativecommons.org/licenses/by/4.0/). 